\title{
Keeping that youthful look
}

\section{Sean C. Solomon}

THE record of impact cratering on Venus, recently revealed from highresolution radar imaging by the Magellan spacecraft, has been interpreted as indicating that the planet underwent catastrophic global resurfacing about 500 million years ago'. Although other interpretations of the crater characteristics have been suggested ${ }^{2}$, the possibility of geologically rapid global resurfacing on the planet that most resembles our own in terms of mass, density and bulk composition has generated widespread interest: what mechanisms could cause such a catastrophe? Several ideas were discussed at the Fall Meeting of the American Geophysical Union last month*.

The average age of Venus's surface has been estimated from the number of larger impact craters (the planet's thick atmosphere has tended to protect it from the smaller bodies that would give craters smaller than $30 \mathrm{~km}$ in diameter) and from the cratering rate on Earth and the Moon, or from estimates of the number of asteroids whose orbits cross that of Venus $^{3}$. The answer, 500 million years ${ }^{1.2}$ (a tenth of the age of the Solar System), is not in itself remarkable for a planet which, like the Earth, has a hot, dynamic interior - despite the absence of significant erosion. What is remarkable is that the craters at all sizes are indistinguishable from a random population (that is, spatial variations in crater density are not larger than those due to chance) and almost none has been significantly modified by tectonic strain or by volcanic flows beyond the crater $\mathrm{rm}^{1.2}$ (Fig. 1), even though Magellan images show volcanic and tectonic features to be nearly ubiquitous on Venus.

The interpretation of Schaber and

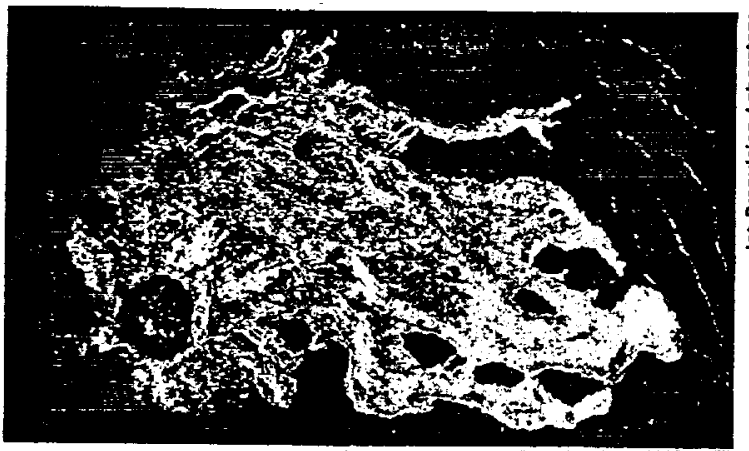

FIG. 1 Magellan radar image of an impact crater on Venus, approximately $70 \mathrm{~km}$ in diameter. Radar-bright, roughtextured ejecta extending up to $60 \mathrm{~km}$ from the crater rim and the remarkable bright flow features extending more than $300 \mathrm{~km}$ from the crater walls are thought to date from the impact ${ }^{1}$ and have not been modified to any significant degree by later deformation or volcanism outside the crater (although the generally radar-dark. smooth floor of the crater may contain younger volcanic deposits). others 1 is that most of the surface dates from the end of a global resurfacing event that ceased about 500 million years ago, and that the small fraction of craters volcanically embayed or modified by deformation indicate that volcanic and tectonic activity has since been at much lower levels. Phillips and colleagues ${ }^{2}$ have argued that a correlation of locations of modified craters with areas of crater density and an inverse correlation between crater density and Magellan radar backscatter (a quantity elevated in regions of high topography and high roughness, both thought to be ignatures of comparative geological youth) indicate that the Venus surface exhibits a spectrum of ages. (The paucity of small craters, however, hampers the use of crater density in determining the relative ages of geological units, a method used with the other solid planets and satellites.) Phillips et al. have described an alternative model in which resurfacing occurs episodically in small ches a few hundred kilometres in extent, with a characteristic time be(Fig. 2).

Nonetheless, the catastrophic hypothesis and its possible mechanisms have captured the lion's share of the attenton. At the meeting, D. L. Turcotte (Cornell University) proposed that global lithospheric overturn operates

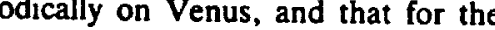
past 500 million years the lithosphere has been cooling and mechanically stable. tion of the lithosphere is not an explicit component of Turcotte's scheme, he argues that a cool and thick lithosphere is in better agreement (than one in steady-state conductive equilibrium) with the large values obtained from topographic profiles for the rigidity of the lithosphere at sites of presumed lithospheric bending ${ }^{4}$ and with the large ratio of long-wavelength gravity anomaly to long-wavelength topographic relief at highland areas ${ }^{5}$.

Parmentier and Hess $^{6}$, following Stevenson and Bittker? $^{7}$ have recently suggested that lithospheric stabilization may occur on Venus because of a decrease in the density of lithospheric mantle following melt extraction. But they add that the cooling

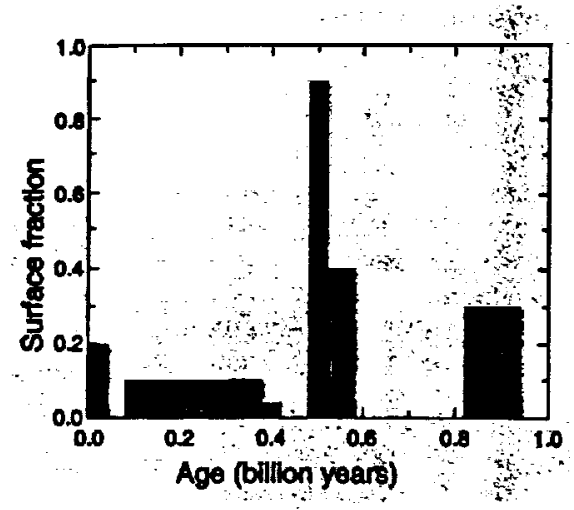

FIG. 2 Two schematic distributions of surface age on Venus. With catastrophic resurfacing ${ }^{2}$ (green histogram), most of the surface is about $\mathbf{5 0 0}$ million years old, but small areas have younger ages. With episodic resurfacing ${ }^{2}$ (red histogram), there is a more widespread distribution of surface ages from 0 to approximately 1,000 million years. Both possibilities are broadly consistent with the distribution and states of presenvation of impact craters on Venus. (Courtesy of R. J. Phillips.)

of such a layer may subsequently raise its density to the point where it becomes unstable. Global overturn of this unstable layer, which would sink back into the mantle, would be followed by partial melting of the upper mantle, global resurfacing, and the gradual development of a new buoyant layer and another extended period of stability. In simple one-dimensional models of this process, the time between lithospheric instability events is $300-500$ million years.

Others point to time-variable mantle convection rather than lithospheric instability as a mechanism for global resurfacing. An early effort to simulate such convection on Venus ${ }^{8}$ led to models in which the characteristic flow speed and mantle heat flux showed large variations (by factors of 2-10) at intervals of $100-$ 200 million years. J. Arkani-Hamed (McGill University) reported improved calculations with better spatial resolution which are still time-varying but which show significantly weaker fluctuations of the order of several tens of a per cent. $V$. Steinbach (Cologne University) drew attention to the potential role of uppermantle phase transitions in governing the radial character of mantle convection in the large terrestrial planets?. He and D. Yuen argue that such phase transitions promote the formation of distinct convecting layers in the upper and lower mantle, but that as the Rayleigh number (governing vigour of flow) in the mantle decreases in response to core cooling, whole-mantle convection tends to become favoured over layered convection. If Venus cooled more rapidly than the Earth early in its history, it may now

-Fall meeting of the American Geophysical Union. Son Francisco, 7-11 December 1992 
have a smaller mantle Rayleigh number and may have gone through a transition to whole-mantle convection, while the Earth may still be characterized by layered mantle flow. Such a transition would have been accompanied by overturn of the upper mantle, the upward transport of heat, and probably by global resurfacing.

The lithospheric instability models as applied to date for Venus suffer from being one-dimensional - global synchroneity is imposed by assumption. Even if such instability mechanisms do operate, it is likely that different parts of the planet will be at different stages in the stabilization and destabilization sequence, and whole-planet temporal variations will be smoothed out by such regional differences. For both lithospheric and mantle-convection mechanisms, attention needs to be given to all the terrestrial planets as a group. Both the Earth and Mars have gone through episodes of great magmatic activity, presumably an indication of variable mantle convective flux, but neither planet shows any evidence of rapid, complete global resurfacing during the past 4,000 million years, and nor do Mercury or the Moon.

Global resurfacing by large-scale overturn of the lithosphere or upper mantle should result in efficient outgassing of the upper mantle; consideration of the ${ }^{0} \mathrm{Ar}$ abundance of Venus's atmosphere suggests that any such widespread outgassing was restricted to times much earlier than 500 million years ago 10 .

Finally, remember that there are alternatives to the catastrophic model that seem to be equally consistent with the characteristics of impact craters on Venus ${ }^{2}$. Further study of the Magellan images of impact features and continued development of dynamic models of the planet's interior should sharpen the competing hypotheses and may lead to an improved understanding of mantle convection and melting on all of the terrestrial planets, including the Earth.

Sean C. Solomon is in the Department of Terrestrial Magnetism, Carnegie Institution of Washington, Washington DC 20015, USA.

1. Schuber, G. G. et al. J. Geophys. Ros. 97. 1325713501 (1992)

2. Phillips, R. J. et al. J. Goophys. Aus. it. 15923

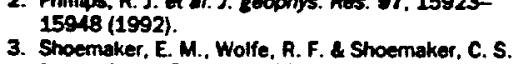
Lunar planot. Sci. 22. 1253-1254 (1991).

4. Sandwell, D. T. \& Schubert, G. J. geophys. Res. 97. 16069-16083 (1992).

5. Smrekar. S. E. \& Phillips, A. J. Earth planet. Scl. Lett. 107, 582-597 (1991).

6. Parmentier, E. M. 4 Hess, P. C. Geophys. Res. Lett. 19, 2015-2018 (1992).

7. Sievenson, D. J. \& Bitther, S. 5. Lunar planet. Sci. 21. $1200-1201$ (1990)

8. Arkani-Hamed. J. \& Toksöz. M. N. Phys. Earth planet inter. 34, 232-250 (1984).

9. Steinbach. V. Yuen, D. A. Geophys. Res. Lett. 19. 2243-2246 (1992).

10. Matsui. T. \& Tajiks. E. Lunar planet. Sci. 22.863 (1991). 